\title{
Association of Triglyceride Glucose Index and Risk of Cardiovascular Disease in Female Health Colleges
}

\author{
DARA AL-DISI, Ph.D. \\ The Department of Community Health Sciences, College of Applied Medical Sciences, King Saud University, Riyadh 11451, \\ Saudi Arabia
}

\begin{abstract}
Background: Cardiovascular disease (CVD) is projected to be responsible for millions of deaths worldwide annually, including Saudi Arabia. CVD risk factors are highly prevalent, particularly among women in Saudi Arabia. Early awareness and treatment of cardiovascular signs before a CVD event occurs are therefore critical. Increased triglycerides and fasting glucose levels are two important risks for CVD, and the triglyceride glucose (TyG) index has been proved to predict CVD in many studies.
\end{abstract}

Aim of Study: This study aims to examine the association of TyG-index and risk of cardiovascular disease in female health colleges to identify individuals at risk of developing CVD events.

Methods: A total of 128 females from female health colleges in Riyadh participated in this cross-sectional study. Glucose and lipid profile parameters along with anthropometric and central obesity parameters were measured. The TyG index and the association between the TyG index and anthropometric and lipid profile parameters using the Spearman correlation coefficient were calculated. ROC analysis was used to detect long-term cardiovascular risk among women by using the TyG index against standard Framingham and ASVCVD risk scores.

Results: This study identified that TyG-index was associated with BMI and waist circumference, which are wellestablished anthropometric risk factors for CVD progression, along with total-cholesterol. A significant positive but weak association between TyG index and Framingham risk scores and lifetime ASCVD score was found. However, the TyG index may not be independently used to identify the people at risk of developing CVD as observed by the low area under the curves (AUC) scores using standard Framingham risk scores and ASCVD risk scores as gold standards.

Conclusion: In conclusion, the positive correlation between TyG index and anthropometric indices and total cholesterol, which are known cardiovascular risks, suggests that the TyG index might be a useful indicator for early identification of CVD in conjunction with other established instruments, such as Farmington and lifetime ASCVD risk scores.

Correspondence to: Dr. Dara Al-Disi, E-Mail: daldisi@ksu.edu.sa
Key Words: TyG index - Insulin Resistance - CVD - Saudi women.

\section{Introduction}

CARDIOVASCULAR disease (CVD) is projected to be responsible for 23 million deaths worldwide annually by 2030 . CVD has also become an important health concern in the Gulf Council countries, including Saudi Arabia, where more than $45 \%$ of all deaths are attributed to CVD [1-3]. In particular, CVD risk factors are highly prevalent among women in Saudi Arabia [4]. The PURE-Saudi study also revealed that CVD risk factors and unhealthy lifestyles are highly prevalent among Saudi adult populations. Along with the well-known CVD risk factors, the PURE-Saudi study also revealed that women had higher self-reported sadness, several periods of stress, and a permanent feeling of stress [1]. Gazzaz et al. (2018) reported high-level stress in medical college students among Saudi students, where the primary stress source was academic [5] Burnout and stress among medical staff and students are common, with likely severe professional and personal effects; there is substantial evidence that cardiovascular health is adversely affected by academic stress [6,7].

Awareness of CVD and its risk factors among the population is crucial to early identification and intervention. Al-Baghli et al. (2010) estimated cardiovascular disease awareness (CVD) in a screening campaign in the eastern province of Saudi Arabia and reported that only $2.7 \%$ of the participants were aware of having cardiovascular disease [8]. Usually, those who are unaware will remain as such until a CVD event occurs. By then, substantial harm or disability will have occurred. One of the early manifestations of CHD in individuals at risk is acute myocardial infarction or sudden 
death [9]. Early awareness and treatment of cardiovascular problems before a CVD event are therefore critical.

Researchers and clinicians have developed and utilized various tools to measure cardiovascular risk to identify individuals with risk factors requiring early treatment and those who are not at risk or low risk [10]. Increased triglycerides and fasting glucose levels are two critical components of metabolic syndrome, and they are the most important risk factor for CVD. The triglyceride glucose (TyG) index has been proved to predict CVD in many studies. It is a simple surrogate estimate of insulin resistance and has recently been shown to predict CVD disease in the general population. Researchers have also proposed the TyG index to be used as an independent biomarker of atherosclerosis in CVD patients [11]. However, this was not confirmed in the study conducted by Cho et al. (2013) [12]. Therefore, there is a need for further studies assessing the accuracy of the TyG index in identifying individuals at risk of developing CAD. This study attempts to assess the role of the TyG index in identifying individuals at risk of CVD using Framingham 30-year (FS30) risk score and the 2013 American Heart Association/American College's atherosclerotic cardiovascular disease (ASCVD) risk score.

\section{Subjects and Methods}

\section{Study population:}

The participants for this cross-sectional study were recruited from the female section of The Applied Medical Science College, central plaza, and the medical city in Riyadh, Saudi Arabia. Recruitment involved distributing recruitment materials (ads, posters, flyers) at various sites of King Saud University, Medical city, and the central plaza between $31 / 8 / 2014$ to $12 / 1 / 2016$. A total of 128 females with an age range of 19 to 56 participated in this study. Subjects with a history of CVD, cancer, severe disability, or severe medical conditions were excluded from this study. The College of Applied Medical Sciences' research ethics committee approved the study protocol. This study followed the Helsinki declaration principle.

\section{Anthropometric measures and blood analysis:}

Anthropometric measurements and blood withdrawal were conducted in the primary health care centers following overnight fasting of at least 10 hours. Anthropometric measurements included weight, height, waist circumference along with mean diastolic and systolic blood pressure.

\section{Biochemical analysis:}

Fasting lipids and glucose were measured using a chemical analyzer (Konelab, Espoo, Finland). The TyG index was calculated by the formula $\ln$ [fasting triglycerides $(\mathrm{mg} / \mathrm{dL}) \mathrm{x}$ fasting glucose $(\mathrm{mg} / \mathrm{dL}) / 2][10]$.

\section{Cardiovascular risk scoring:}

This study calculated CVD risk scores using the most widely used risk equations, including the Framingham 30-year risk score (FS30) and lifetime predicted risk of atherosclerotic cardiovascular disease (ASCVD). Framingham's risk scoring was used with four variations. First, Framingham's 30year Hard CVD score is based on BMI (FS30 BMI Hard CVD) risk score, where hard CVD means acute myocardial infarction, death from coronary artery disease, and stroke. The second was Framingham's 30-year risk score based on the lipid profile of hard cardiovascular disease (FS30 Lipid Hard CVD). Third, Framingham's 30-year BMI risk score for full cardiovascular disease or other incidents, such as coronary insufficiency, angina pectoris, transient ischaemic assault (FS30 BMI Full CVD). Fourth, a 30-year Framingham risk score based on the full cardiovascular disease lipid profile (FS30 Lipid Full CVD).

Furthermore, the 2013 American Heart Association/American College of Cardiology developed a tool to estimate the 10-year and lifetime predicted risk of atherosclerotic cardiovascular disease (ASCVD) $(13,14)$. For FS30, participants with scores $<12 \%$ were classified as of low risk of CVD, and $>12 \%$ was defined as high risk. For lifetime ASCVD, female participants with $8 \%$ risk were classified as having a high CVD risk [13]

\section{Statistical analysis:}

Data were analyzed using SPSS version 21.0. Mean and the standard deviation was used as descriptive statistics for continuous variables, frequencies, and percentages for categorical variables. Statistical differences in TyG index tertiles were obtained using analysis of variance (ANOVA) and the Kruskal-Wallis test for normal and non-normal data, respectively. Association between TyG index and categorical variables was tested using the chisquare test. Furthermore, the Spearman correlation coefficient was used to determine the association between the TyG index and other risk scores. The area under the curves (AUCs) for the TyG index was obtained using ROC analysis. $p<0.05$ was considered significant. 


\section{Results}

A total of 128 females (age 19-56 yrs) participated in this study. Table (1) shows the descriptive statistics of study parameters. The participants' average age was $31.8 \pm 8.4$, and the average BMI was $28.3 \pm 5.3$. The average TyG index calculated was 4.6 \pm 0.3 . The average hard CVD FS30 based on lipids and BMI were $2.4 \pm 2.6$ and $3.5 \pm 3.5$, whereas average full CVD FS30 based on lipids and BMI were $5.5 \pm 5.1$ and $7.4 \pm 6.3$ respectively. The average Lifetime ASCVD risk was $17.5 \pm 13.3$. Table (1) also reports the results of the study parameters according to TyG index tertiles. The mean age, BMI, and WHR of the participants were significantly higher in the 3 rd tertile than the ${ }^{1 \text { st }}$ tertile. Mean $\mathrm{BMI}$ was also higher in the second tertile as compared to the first tertile. Furthermore, mean glucose and mean total cholesterol were also significantly higher in the 3 rd tertile as compared to the 1 st and second tertile. The mean hard and full CVD FS30 based on lipids and lifetime ASCVD risk was significantly higher in the third tertile as compared to the first and second tertile. No differences in TyG index tertiles were observed in the mean of hard and full CVD FS30 based on BMI; however, there was an increasing but insignificant trend.

Table (2) shows the relationship between the TyG index and study parameters using the Spear- man correlation coefficient. TyG index was significantly and positively associated with BMI and total cholesterol with Spearman correlation coefficients of $0.24(p<0.01)$ and $0.32(p<0.01)$, respectively. The Spearman correlation coefficient between TyG index and hard CVD FS30 based on lipids, hard CVD FS30 based on BMI, full CVD FS30 based on lipids, full CVD FS30 based on $\mathrm{BMI}$ and lifetime ASCVD risk were $0.28(p<0.01)$, $0.20(p<0.05), 0.25(p<0.01), 0.20(p<0.05)$ and $0.28(p<0.01)$ respectively. The correlation between the TyG index and four Framingham and lifetime ASCVD scores was between 0.2-0.28, which is regarded as weak.

Table (3) presents the ROC analysis to detect the long-term cardiovascular risk among women by using the TyG index against standard Framingham and ASVCVD risk scores. The areas under the curve (AUC) of the ROC plots were 0.49, 0.60, and 0.58 for TyG index using the FS30 BMI hard CVD, FS30 BMI Full CVD, and FS30 lipids full CVID. None of them were significant, as indicated by $p$-values which were all greater than 0.05 . The area under the curve of the ROC plot for the TyG index against lifetime ASCVD risk was 0.66, which was significant. Furthermore, all these AUCs for the TyG index achieved low sensitivities and specificities.

Table (1): Descriptive statistics according to TyG index Tertiles.

\begin{tabular}{|c|c|c|c|c|c|}
\hline \multirow{2}{*}{ Parameters } & \multicolumn{5}{|c|}{ TyG Tertiles } \\
\hline & Overall & $1 \mathrm{st}(\leq 4.5)$ & 2nd (4.6-4.7) & $3 \mathrm{rd}(\geq 4.8)$ & $p$-values \\
\hline Age (Years) & $31.8 \pm 8.4$ & $30.0 \pm 7.9$ & $31.1 \pm 7.7$ & $34.5 \pm 9.0^{\mathbf{A}}$ & 0.039 \\
\hline BMI $\left(\mathrm{kg} / \mathrm{m}^{2}\right)$ & $28.3 \pm 5.3$ & $26.4 \pm 5.2$ & $28.9 \pm 4.9 \mathrm{~A}$ & $29.6 \pm 5.5 \mathrm{~A}$ & 0.015 \\
\hline $\mathrm{WC}(\mathrm{cm})$ & $83.2 \pm 11.2$ & $79.4 \pm 10.9$ & $82.8 \pm 10.4$ & $87.3 \pm 11.2 \mathrm{~A}$ & 0.005 \\
\hline $\mathrm{SBP}(\mathrm{mmHg})$ & $101.3 \pm 12.5$ & $98.5 \pm 9.3$ & $103.2 \pm 14.4$ & $102.1 \pm 13.0$ & 0.19 \\
\hline $\mathrm{DPP}(\mathrm{mmHg})$ & $71.2 \pm 10.4$ & $69.0 \pm 7.7$ & $72.8 \pm 10.6$ & $71.6 \pm 12.3$ & 0.21 \\
\hline glucose (mg/dL) & $99.1 \pm 17.1$ & $94.1 \pm 12.9$ & $96.6 \pm 10.6$ & $107.0 \pm 23.2 \mathbf{A B}$ & 0.001 \\
\hline T-Cholesterol (mg/dL) & $161.9 \pm 38.8$ & $147.3 \pm 27.4$ & $154.8 \pm 32.5$ & $184.6 \pm 45.2^{\mathbf{A B}}$ & $<0.001$ \\
\hline HDL (mg/dL) & $55.6 \pm 16.1$ & $53.7 \pm 15.1$ & $55.1 \pm 15.7$ & $58.0 \pm 17.7$ & 0.47 \\
\hline LDL (mg/dL) & $81.7 \pm 34.4$ & $79.8 \pm 22.9$ & $78.8 \pm 27.7$ & $87.0 \pm 48.4$ & 0.49 \\
\hline TG (mg/dL) & $122.8 \pm 70.8$ & $68.8 \pm 15.4$ & $104.7 \pm 18.3 \mathbf{A}$ & $197.9 \pm 77.9^{\mathbf{A B}}$ & $<0.001$ \\
\hline TyG Index & $4.6 \pm 0.3$ & $4.4 \pm 0.1$ & $4.6 \pm 0.1 \mathrm{~A}$ & $4.9 \pm 0.2^{\mathbf{A B}}$ & $<0.001$ \\
\hline FS30 Lipids Hard CVD & $2.4 \pm 2.6$ & $1.7 \pm 2.0$ & $2.0 \pm 1.8$ & $3.5 \pm 3.5^{\mathbf{A B}}$ & 0.003 \\
\hline FS30 BMI Hard CVD & $3.5 \pm 3.5$ & $2.9 \pm 3.5$ & $3.4 \pm 3.2$ & $4.4 \pm 3.7$ & 0.159 \\
\hline FS30 BMI Full CVD & $7.4 \pm 6.3$ & $6.1 \pm 6.3$ & $7.1 \pm 5.7$ & $9.0 \pm 6.8$ & 0.101 \\
\hline FS30 Lipids Full CVD & $5.5 \pm 5.1$ & $4.2 \pm 4.1$ & $4.8 \pm 3.5$ & $7.6 \pm 6.8^{\mathbf{A B}}$ & 0.005 \\
\hline Life time ASCVD risk & $17.5 \pm 13.3$ & $12.9 \pm 11.0$ & $16.5 \pm 12.6$ & $23.3 \pm 14.4 \mathbf{A B}$ & 0.001 \\
\hline Smoking (Yes) & $2(1.6)$ & $1(2.4)$ & $1(2.2)$ & $0(0.0)$ & 0.62 \\
\hline Hypertension (Yes) & $3(3.1)$ & $0(0.0)$ & $2(6.9)$ & $1(2.9)$ & 0.30 \\
\hline Dyslipidemia (Yes) & $7(10.6)$ & $0(0.0)$ & $4(19.0)$ & $3(13.0)$ & 0.12 \\
\hline Diabetes (Yes) & $1(.9)$ & $1(2.8)$ & $0(0.0)$ & $0(0.0)$ & 0.37 \\
\hline Family History of CVD (Yes) & $24(19.4)$ & $10(23.8)$ & $8(18.2)$ & $6(15.8)$ & 0.64 \\
\hline
\end{tabular}

Note: Data presented as mean \pm SD for continuous variables and frequency (\%) for categorical variables. $p<0.05$ is considered significant. 
Table (2): Correlations matrix between TyG Index and select parameters.

\begin{tabular}{|c|c|c|c|c|c|c|c|c|c|}
\hline Parameters & TyG & HDL & LDL & $\mathrm{TC}$ & BMI & HCVD-L & $\begin{array}{c}\text { HCVD- } \\
\text { BMI }\end{array}$ & $\begin{array}{c}\text { FCVD- } \\
\text { BMI }\end{array}$ & $\begin{array}{l}\text { FCVD- } \\
\text { Lipids }\end{array}$ \\
\hline HDL (mg/dL) & -0.01 & & & & & & & & \\
\hline $\mathrm{LDL}(\mathrm{mg} / \mathrm{dL})$ & 0.01 & 0.01 & & & & & & & \\
\hline $\mathrm{TC}(\mathrm{mg} / \mathrm{dL})$ & $0.32 * *$ & $0.41 * *$ & $0.81 * *$ & & & & & & \\
\hline $\operatorname{BMI}\left(\mathrm{kg} / \mathrm{m}^{2}\right)$ & $0.24 * *$ & -0.08 & 0.09 & 0.11 & & & & & \\
\hline FS30 Lipids Hard CVD & $0.28 * *$ & -0.02 & $0.52 * *$ & $0.51 * *$ & $0.40 * *$ & & & & \\
\hline FS30 BMI Hard CVD & $0.20^{*}$ & 0.06 & $0.27 * *$ & $0.31 * *$ & $0.59 * *$ & $0.88 * *$ & & & \\
\hline FS30 BMI Full CVD & $0.20 *$ & 0.05 & $0.24 * *$ & $0.27 * *$ & $0.58 * *$ & $0.87 * *$ & $0.97 * *$ & & \\
\hline FS30 Lipids Full CVD & $0.25 * *$ & -0.08 & $0.45 * *$ & $0.41 * *$ & $0.42 * *$ & $0.95 * *$ & $0.92 * *$ & $0.92 * *$ & \\
\hline Life time ASCVD risk & $0.28 * *$ & $0.20 *$ & $0.62 * *$ & $0.66 * *$ & 0.05 & $0.49 * *$ & $0.31 * *$ & $0.30 * *$ & $0.431 * *$ \\
\hline
\end{tabular}

Note: Data presented as spearman correlation coefficient. ** \&* Indicates significance at 0.01 and 0.05 level.

Table (3): The area under the curve (AUC) of TyG-index in detecting the long-term cardiovascular risk among women, using different risk scores.

\begin{tabular}{|c|c|c|c|c|c|c|}
\hline \multirow{2}{*}{ FS30 and ASCVD index } & \multirow{2}{*}{$\begin{array}{l}\text { Patients } \\
\text { at CVD risk }\end{array}$} & \multicolumn{5}{|c|}{ TyG-Index } \\
\hline & & $\mathrm{AUC} \pm \mathrm{SE}$ & $p$-values & Cut-off & Sensitivity & Specificity \\
\hline FS30 BMI Hard CVD & $3(2.3)$ & $0.49 \pm 0.17$ & 0.956 & 4.56 & $67 \%$ & $44 \%$ \\
\hline FS30 BMI Full CVD & $23(18.0)$ & $0.60 \pm 0.07$ & 0.155 & 4.59 & $56 \%$ & $54 \%$ \\
\hline FS30 Lipids Full CVD & $13(10.2)$ & $0.58 \pm 0.09$ & 0.346 & 4.59 & $54 \%$ & $53 \%$ \\
\hline Life time ASCVD risk & $46(35.9)$ & $0.66 \pm 0.05$ & 0.003 & 4.59 & $63 \%$ & $61 \%$ \\
\hline
\end{tabular}

Note: Patients were considered at CVD risk if either 30-year Framingham risk score or lifetime ASCVD score were $<12 \%$ and $8 \%$, respectively.

\section{Discussion}

The TyG index has been used to quantify insulin resistance, which compares triglyceride and glucose concentrations, and is a standard assay of insulin resistance. This study identified that TyG-index was associated with BMI and waist circumference, which are well-established anthropometric risk factors for CVD progression, along with totalcholesterol. Furthermore, there was a significant positive but weak association between TyG index and Framingham risk scores and lifetime ASCVD score. However, the TyG index may not be independently used to identify the people at risk of developing CVD, identified by AUC scores using standard Framingham risk scores and ASCVD risk scores as gold standards. The areas under the curve (AUC) were $0.49,0.60$, and 0.58 for TyG index using the FS30 BMI hard CVD, FS30 BMI Full CVD, and FS30 lipids full CVD. None of them were significant, as indicated by $p$-values which were all greater than 0.05 . The area under the curve for TyG index against lifetime ASCVD risk was 0.66 , which was significant. However, all these AUCs for the TyG index achieved low sensitivities and specificities.

Recent studies have shown that a higher risk of adverse cardiac events can be predicted by a higher TyG index $[\mathbf{1 5}, \mathbf{1 6}]$. In patients with stable coronary artery disease, Jin et al. (2018) have also found that TyG can indicate clinical outcomes [15]. Some studies have shown an association between Atherosclerotic cardiovascular disease and TyG index [17] A 10-year follow-up study with 5014 patients revealed a higher risk by lifetime ASCVD and is positively associated with a higher TyG index. The index could also give the Framingham risk score additive value for ASCVD prediction [18]. These results were consistent with our study's findings, which observed positive but weak associations with lifetime ASCVD and Framingham risk scores. This weak relationship might result from lower TyG index values in the participants with a maximum value of 5.3. Li et al. (2019) reported that TyG-index is a better predictor for CVD events in women than in men, where TyG is more than 9.53 [19]. However, the authors did not ponder how gender differences can modulate the effects of TyG on cardiovascular risks. However, our study failed to establish the TyG index as an independent biomarker of cardiovascular disease in women, which contrasts with the Korean study findings, with 12,326 participants showing that the TyG index is an independent biomarker for identifying coronary artery calcification [20]. Kim et al. (2017) also found that coronary artery athero- 
sclerosis was independently associated with TyG index in healthy Korean adults [21].

This study also used TyG index tertile (increasing $\mathrm{TyG}$ ) to assess the relationship between tertiles and study parameters. Significant differences were observed in BMI, waist circumference, glucose, triglycerides, and total cholesterol. Researchers have reported mixed results for the relationship between anthropometric indices and cardiovascular risk [22]. Increased waist circumference and BMI have shown to be indicative of higher coronary risk $[19,22,23]$. Although $\mathrm{BMI}$ is a well-known risk factor for T2DM and CVD development, cardiovascular risk factors such as metabolic syndrome, hyperglycemia, hypertension, and hypertriglyceridemia have been more closely associated with WHR than with WC or BMI, especially in the Asian population $[\mathbf{2 4 , 2 5 ]}$. Although this study did not include WHR, TyG-index has shown a significant positive association with $\mathrm{WC}, \mathrm{BMI}$, and metabolic components, consistent with the findings reported by previous studies $[19,22,23]$.

There were some limitations in this study. First, the nature of this study was cross-sectional; therefore, causality can not be established. Secondly, the sample size was small. Third, this study did not include the waist-to-hip ratio because of the absence of hip circumference measurements, which is a significant CVD risk factor.

In conclusion, the positive correlation between TyG index and anthropometric indices and total cholesterol, which are known cardiovascular risks, suggests that the TyG index might provide useful information to identify patients at risk of developing CVD. This role is further established by positive correlations between TyG-index and Framingham risk scores and lifetime ASCVD score. However, this study does not validate TyG-index to be independently used to identify the people at risk of developing CVD, as shown by low AUC scores using standard Framingham risk scores and lifetime ASCVD risk scores as gold standards.

Acknowledgments: The author thanks all the subjects who participated in this study.

Conflicts of interest: The author declares no conflicts of interest.

\section{References}

1- ALHABIB K.F., BATAIS M.A., ALMIGBAL T.H., ALSHAMIRI M.Q., ALTARADI H., RANGARAJAN S., et al.: Demographic, behavioral, and cardiovascular disease risk factors in the Saudi population: Results from the Prospective Urban Rural Epidemiology study (PURESaudi). BMC public health, 20 (1): 1-14, 2020.
2- ALJEFREE N. and AHMED F.: Prevalence of cardiovascular disease and associated risk factors among adult population in the Gulf region: A systematic review. Advances in Public Health, 2015, 2015.

3- WHO. Cardiovascular diseases (CVDs) fact sheet. Geneva, 2021.

4- ALSHAIKH M.K., FILIPPIDIS F.T., BALDOVE J.P., MAJEED A. and RAWAF S.: Women in Saudi Arabia and the prevalence of cardiovascular risk factors: A systematic review. Journal of environmental and public health, 2016.

5- GAZZAZ Z.J., BAIG M., AL ALHENDI B.S.M., AL SULIMAN M.M.O., AL ALHENDI A.S., AL-GRAD M.S.H., et al.: Perceived stress, reasons for and sources of stress among medical students at Rabigh Medical College, King Abdulaziz University, Jeddah, Saudi Arabia. BMC Medical Education, 18 (1): 1-9, 2018.

6- AHANEKU J.E., NWOSU C.M. and AHANEKU G.I.: Academic stress and cardiovascular health. Academic Medicine, 75 (6): 567-8, 2000.

7- ELWESS N.L. and VOGT F.D.: Heart Rate and Stress in a College Setting. Bioscene: Journal of College Biology Teaching, 31 (4): 20-3, 2005.

8- AL-BAGHLI N.A., AL-GHAMDI A.J., AL-TURKI K.A., EL-ZUBAIER A.G., AL-MOSTAFA B.A., AL-BAGHLI F.A., et al.: Awareness of cardiovascular disease in eastern Saudi Arabia. Journal of Family and Community Medicine, 17 (1): 15, 2010.

9- SANCHIS-GOMAR F., PEREZ-QUILIS C., LEISCHIK R. and LUCIA A.: Epidemiology of coronary heart disease and acute coronary syndrome. Annals of Translational Medicine, 4 (13), 2016.

10- LLOYD-JONES D.M., BRAUN L.T., NDUMELE C.E., SMITH JR S.C., SPERLING L.S., VIRANI S.S., et al.: Use of risk assessment tools to guide decision-making in the primary prevention of atherosclerotic cardiovascular disease: A special report from the American Heart Association and American College of Cardiology. Circulation, 139 (25): e1162-e77, 2019.

11- LEE E.Y., YANG H.K., LEE J., KANG B., YANG Y., LEE S.H., et al.: Triglyceride glucose index, a marker of insulin resistance, is associated with coronary artery stenosis in asymptomatic subjects with type 2 diabetes. Lipids in Health and Disease, 15 (1): 1-7, 2016.

12- CHO Y.R., ANN S.H., WON K.B., PARK G.M., KIM Y.G., YANG D.H., et al.: Association between insulin resistance, hyperglycemia, and coronary artery disease according to the presence of diabetes. Scientific Reports, 9 (1): 1-7, 2019.

13- ABULMEATY M.M., ALMAJWAL A.M., ALMADANI N.K., ALDOSARI M.S., ALNAJIM A.A., ALI S.B., et al.: Anthropometric and central obesity indices as predictors of long-term cardiometabolic risk among Saudi young and middle-aged men and women. Saudi Medical Journal, 38 (4): 372, 2017.

14- GUERRA-SILVA N.M.M., SANTUCCI F.S., MOREIRA R.C., TASHIMA C.M., DE MELO S.C.C.S., PEREIRA L.R.L, et al.: Coronary disease risk assessment in men: Comparison between ASCVD Risk versus Framingham. International Journal of Cardiology, 228: 481-7, 2017. 
15- JIN J.L., CAO Y.X., WU L.G., YOU X.D., GUO Y.L., WU N. Q., et al.: Triglyceride glucose index for predicting cardiovascular outcomes in patients with coronary artery disease. Journal of Thoracic Disease, 10 (11): 6137, 2018.

16- LIU Y., WU M., XU J., SHA D., XU B. and KANG L.: Association between Triglyceride and glycose (TyG) index and subclinical myocardial injury. Nutrition, Metabolism and Cardiovascular Diseases, 30 (11): 2072-6, 2020.

17- HONG S., HAN K. and PARK C.Y.: The triglyceride glucose index is a simple and low-cost marker associated with atherosclerotic cardiovascular disease: A populationbased study. BMC Medicine, 18 (1): 1-8, 2020.

18- SÁNCHEZ-ÍNIIGO L., NAVARRO-GONZÁLEZ D., FERNÁNDEZ-MONTERO A., PASTRANA-DELGADO J. and MARTÍNEZ J.A.: The TyG index may predict the development of cardiovascular events. European Journal of Clinical Investigation, 46 (2): 189-97, 2016.

19- LI S., GUO B., CHEN H., SHI Z., LI Y., TIAN Q., et al.: The role of the triglyceride (triacylglycerol) glucose index in the development of cardiovascular events: A retrospective cohort analysis. Scientific Reports, 9 (1): 1-9, 2019.

20- WON K.B., PARK E.J., HAN D., LEE J.H., CHOI S.Y., CHUN E.J., et al.: Triglyceride glucose index is an independent predictor for the progression of coronary artery calcification in the absence of heavy coronary artery calcification at baseline. Cardiovascular Diabetology, 19 (1): 1-8, 2020.

21- KIM M.K., AHN C.W., KANG S., NAM J.S., KIM K.R. and PARK J.S.: Relationship between the triglyceride glucose index and coronary artery calcification in Korean adults. Cardiovascular Diabetology, 16 (1): 1-7, 2017.

22- GELBER R.P., GAZIANO J.M., ORAV E.J., MANSON J.E., BURING J.E. and KURTH T.: Measures of obesity and cardiovascular risk among men and women. Journal of the American College of Cardiology, 52 (8): 605-15, 2008.

23- REXRODE K.M., CAREY V.J., HENNEKENS C.H., WALTERS E.E., COLDITZ G.A., STAMPFER M.J., et al.: Abdominal adiposity and coronary heart disease in women. Jama, 280 (21): 1843-8, 1998.

24- ESMAILLZADEH A., MIRMIRAN P. and AZIZI F.: Comparative evaluation of anthropometric measures to predict cardiovascular risk factors in Tehranian adult women. Public Health Nutrition, 9 (1): 61-9, 2006.

25- HSIEH S.D. and MUTO T.: The superiority of waist-toheight ratio as an anthropometric index to evaluate clustering of coronary risk factors among non-obese men and women. Preventive Medicine, 40 (2): 216-20, 2005. 


\section{ارتباط مؤثر الجلوكوز ثلاثى الجليسريل وخطر الإصابة

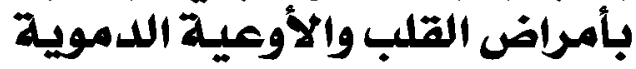

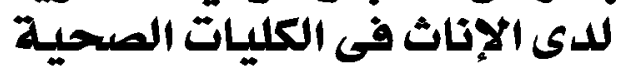

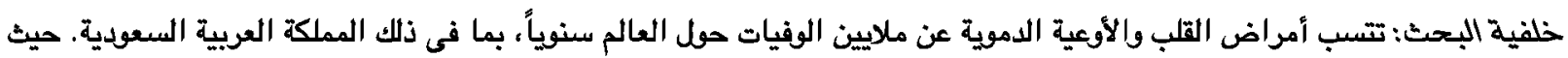

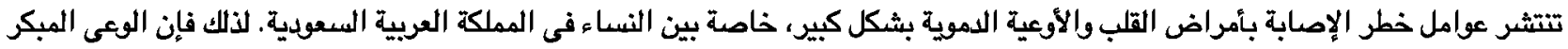

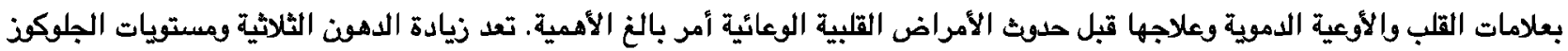

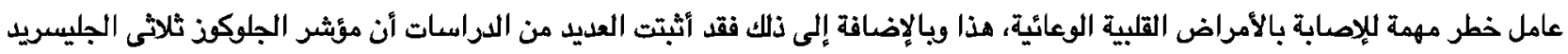
(TyG)

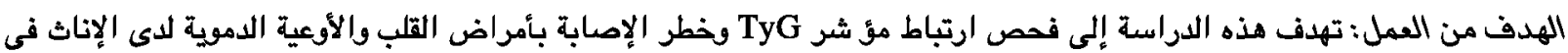

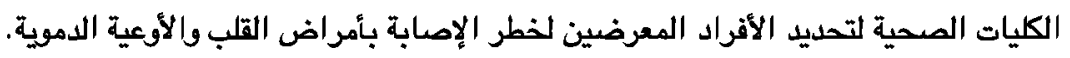

المواد والأساليب: ثارك عدد ^rا طالبة من الكيات الصحية بالرياض فى هذه الدراسة المقطعية. تم قياس مؤشرات الجلوكوذ والدهون

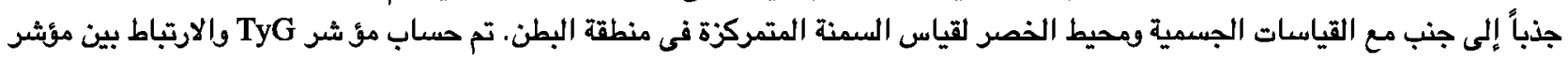

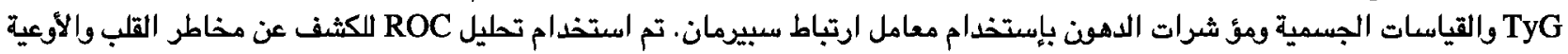
الدموية على المدى الطويل بين الإناث بإستخدام مؤشر TyG مقابل درجات مخاطر Framingham القياسية و

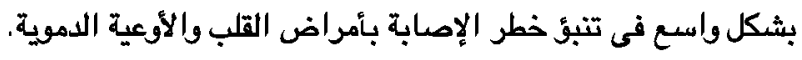

النتائج: حددت هذه الدراسة أن مؤثر TyG كان مرتبطاً بمؤثر كتة الجسم ومحيط الخصر ومستقى الكوليسترول الكلى، وهما من عوامل

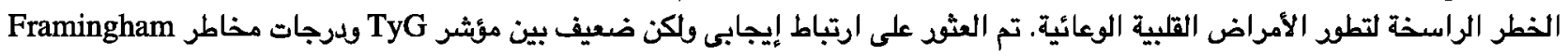

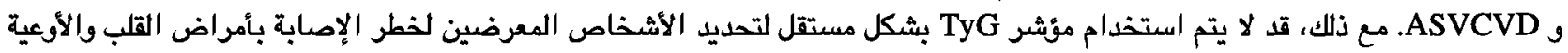

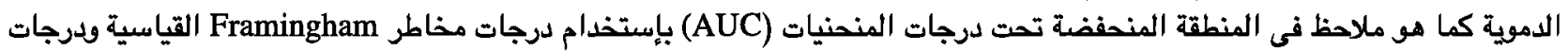

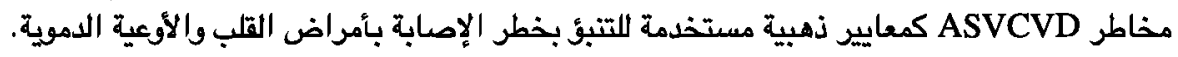

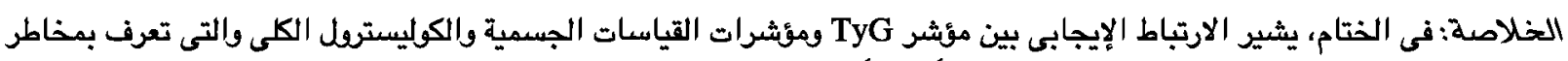

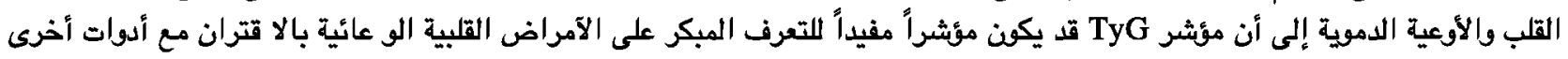
ثابتة، مثل 